Infográfico

\title{
Avaliação do paciente com acidente vascular encefálico na era da COVID-19 - inovações no exame neurológico observacional
}

\author{
Eduardo Martins Leal', Gabriel de Deus Vieira', Mayara dos Santos Morais ${ }^{1}$, Isabela Zanellato Marques ${ }^{1}$, \\ Adaucto Wanderley da Nóbrega Junior ${ }^{1}$, Gisele Espíndola', Katia Lin ${ }^{1}$
}

1. Programa de Residência Médica em Neurologia, Comissão de Residência Médica (COREME), Hospital Universitário Polydoro Ernani de São Thiago, Universidade Federal de Santa Catarina (HU/UFSC), Campus Professor João David Ferreira Lima. Florianópolis, SC, Brasil.

\begin{abstract}
RESUMO
É objetivo deste projeto apresentar um vídeo ( https://youtu.be/5Sh1PnpeKmk ) demonstrando o exame neurológico observacional na era da COVID-19.
\end{abstract}

DOI: https://doi.org/10.32963/bcmufsc.v6i2.4400

Indexadores: COVID-19; Acidente Vascular Cerebral (AVC); Exame neurológico; Semiologia; Propedêutica Submetido em 7/9/2020; aceito para publicação em 17/10/2020.

Os autores não possuem conflitos de interesse referentes ao presente trabalho. Apoio: CNPq (Conselho Nacional de Desenvolvimento Científico e Tecnológico) Autor para contato: Profa. Katia Lin. E-mail: katia.lin@ufsc.br

A pandemia de 'Coronavirus disease' 2019 (COVID-19) vem impondo mudanças radicais nos diversos sistemas de saúde, na estrutura médicohospitalar, até no relacionamento médico-paciente. Medidas extremas visando a contenção da COVID-19 incluem o isolamento de populações (medidas de 'lockdown'), a suspensão do atendimento ambulatorial de rotina e cirurgias eletivas, a conversão de andares inteiros em enfermarias dedicadas à quarentena de casos COVID-19, com importante impacto no manejo de diversas doenças agudas e crônicas'.

Independentemente das medidas acima, a doença encefalovascular continua afetando a vida de milhares de brasileiros, constituindo um dos principais motivos de atendimento emergencial em hospital-geral, exigindo assistência médica continuada e tempo-sensível.

\section{Referências}

1. Zhao J, Rudd A, Liu R. Challenges and potential solutions of stroke care during the coronavirus disease 2019 (COVID19) outbreak. Stroke 2020;51:1356-7.

2. Antoniello D, Nisar T, Kirchoff-Torres KF, Cheng NT, Liberman AL, Labovitz D. Invited commentary: stroke
Com os atuais indícios de que esta pandemia continuará se prolongando globalmente, médicos-neurologistas e de outras especialidades responsáveis pelo atendimento emergencial do acidente vascular encefálico (AVE) estão sob constante risco de exposição ao COVID-19. Visando minimizar esses riscos, mas mantendo a eficiência e acurácia diagnóstica do exame, inovações no exame neurológico emergencial para o paciente com suspeita de AVE foram implementadas em diversos centros de referência para o atendimento de $\mathrm{AVE}^{2}$.

É objetivo deste projeto, portanto, apresentar um vídeo ( https://youtu.be/5Sh1PnpeKmk ) demonstrando o exame neurológico observacional na era da COVID-19, visando extrair o máximo de informações de forma eficiente, rápida, e minimizando os riscos de contaminação para o médico-assistente.

assessment in the COVID-19 era: the observational neurologic exam. Neurology Blogs: COVID-19 (coronavirus) innovations in care delivery. 2020. Disponível em [https://blogs.neurology.org/covid-19-coronavirus/invitedcommentary-stroke-assessment-in-the-covid-19-era-theobservational-neurologicexam/?utm_source=Facebook\&utm_medium=organic $\% 20$ soc ial] 